\title{
Iterative Machine Learning Method for Pore-Back Artifact Mitigation in High Porosity Membrane FIB-SEM Image Segmentation
}

\author{
Joseph Tracey ${ }^{1}$, Sam Sheng Lin ${ }^{2}$, Jasna Jankovic ${ }^{1}$, Aiden $\mathrm{Zhu}^{2}$, Shawn Zhang ${ }^{2}$ \\ 1. Materials Science and Engineering Department, University of Connecticut, Storrs, Connecticut, USA. \\ 2. DigiM Solution LLC, Burlington, Massachusetts, USA.
}

With the advent of modern microscopy methods, there is an increasing need for efficient and robust image segmentation methods. Machine learning has demonstrated the greatest potential, as a trained model that can be applied to large new sets of images, after learning from a limited set of hand or computer aided segmentations. Furthermore, deep learning can treat extremely complicated features, such as pore-back artifacts.

Pore-back artifacts are associated with porous material imaged with focused ion beam scanning electron microscopy (FIB-SEM). Electron signals go behind the imaging plane, and light the back wall of a pore. Depending on the depth of the pore geometry, a gradient of greyscale contrast is often collected in the same pore space, which leads to difficulties in segmentation. The Pore-back artifact problem is most pronounced when the porosity of the sample is high.

In this project, a combination of Random Forest machine learning 0 and deep residual U-net (ResUnet) neural network deep learning 0 was used to segment pore-backs from a FIB-SEM image stack of a membrane sample with $80 \%$ porosity, Figure $2 \mathrm{a}$.

The 3D image stack was first segmented with supervised Random Forest machine learning, with manual corrections to the pore-backs. Selected images from the first segmentation were then used as training sets for a second segmentation with a deep learning model, where a U-net shaped network is combined with residual units replacing the neural units of the network to integrate skip connections into the U-net, preventing the loss of information as the network gets deeper.

To determine the correlation of training efficiency with length scale of primary features, the second neural network-based segmentations were trained with two varying sized training sets from the first segmentation. One training set was selected from the top of the stack in the first segmentation, while the other training set was selected using segmented images indexed evenly throughout the FIB-SEM stack of 600 images. The training set was composed of either 5 or 20 images in each case, resulting in a total of four trials, as summarized in Table 1. The set of 5 distributed images was indexed 120 slides from one another, while the set with 20 distributed images was trained 30 slides apart from one another.

To compare the quality of the network's predictions, accuracy and loss functions were tracked and reported on three selected locations in the stack, as summarized in Table 1 . The preliminary results show that with a training set of 20 images, the predictions made by the network that are close to the initial index are similar to the distributed model in accuracy and loss. But, for higher indices, i.e., Index-434 and Index-598, the predictions made by the distributed model are more accurate on than those made by the model trained on the initial index model.

When a much smaller training set of 5 images is used, however, the initial-model proved more effective throughout the entire distribution of the 3D sample set than the distributed-model. The loss and accuracy both indicate that the initial-index model is better for such a limited training set. 
The results of these experiments indicate that the training sample distribution has a significant impact on prediction quality. Using a wider distribution for the images helps when the network has enough data to learn from. However, having the quality degrade on all factors for the set including 5 images suggests that using a set with increased diversity might have given the network too much variation with too little data. This suggests that whether or not the training data for a ResUnet should be indexed across the sample or together depends on how large the training set is. The results also demonstrate that the augmented ResUnet can be applied to 3D image sets of polymer membranes with very small data sets to provide initial segmentation, and have an accuracy approaching 90\% when the training set is 20 images distributed throughout the dataset for segmentations throughout a very large image-set. Ongoing work also includes further integration of Random Forest machine learning with deep learning, where both the segmentation of the initial training set and the final target set can be automated.

\section{References:}

[1] S. Zhang et al., Microscopy Today 27 (2019). doi:10.1017/S1551929519000026

[2] A Dravid, Microscopy Today 27(1) (2019), p. 18.

\begin{tabular}{|l|l|l|l|l|}
\hline Predicted image & 20 Initial & 20 Distributed & 5 Initial & 5 Distributed \\
\hline Index-47, Loss & 0.442 & 0.425 & 0.786 & 1.18 \\
\hline Index-47, Accuracy & 0.912 & 0.914 & 0.832 & 0.738 \\
\hline Index-434, Loss & .649 & .300 & .771 & 1.038 \\
\hline Index-434, Accuracy & 0.881 & 0.945 & 0.837 & 0.758 \\
\hline Index-598, Loss & 0.764 & 0.613 & 0.908 & 1.246 \\
\hline Index-598, Accuracy & 0.863 & 0.892 & 0.793 & 0.731 \\
\hline
\end{tabular}

Figure 1. Loss and Accuracy results from predictions at varying indices within the 3D FIB-SEM image-set.

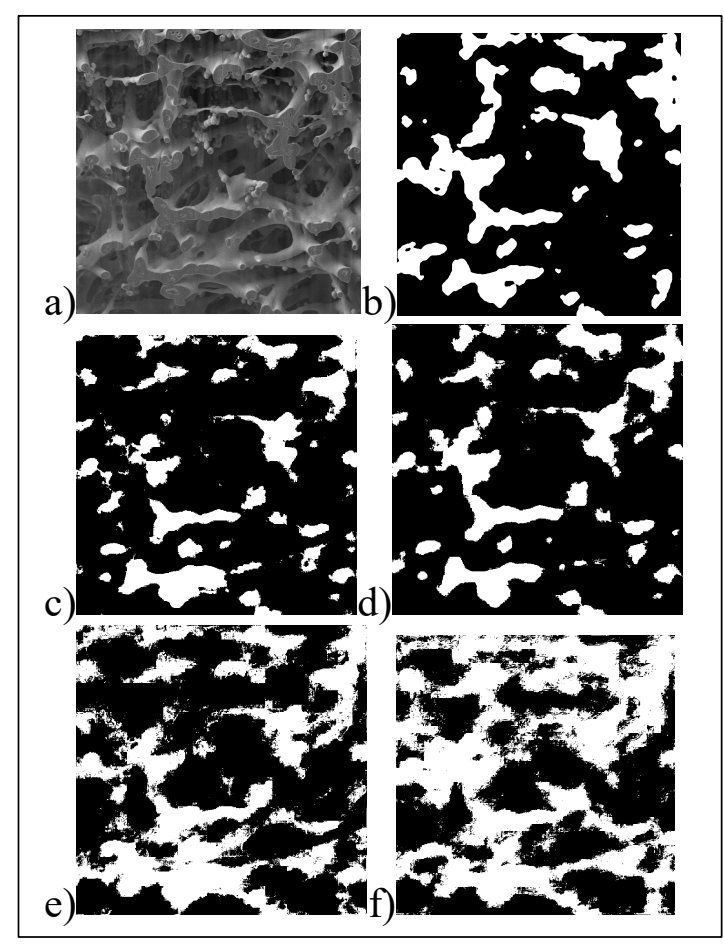

Figure 2. Predictions by Each Model on Index-598. (a) FIBSEM of porous polymer membrane with a porous background. Figures (b)-(f) are 5 segmentations: (b) Ground truth from Random Forest Machine Learning and manual correction; (c) 20 Initial-Model; (d) 20 distributed -Model; (e) 5 Initial Model; (f) 5 Distributed 